P005 LONG-TERM SAFETY AND EFFICACY OF SOLRIAMFETOL FOR EXCESSIVE DAYTIME SLEEPINESS: AN OPEN-LABEL EXTENSION RANDOMISED WITHDRAWAL TRIAL

${ }^{1}$ Colin M Shapiro*, 2,3Geert Mayer, ${ }^{4}$ Jean-Louis Pepin, ${ }^{5}$ Richard Schwab, ${ }^{6}$ Jan Hedner, ${ }^{7}$ Mansor Ahmed, ${ }^{8}$ Nancy Foldvary-Schaefer, ${ }^{9}$ Patrick J Strollo, ${ }^{10}$ Kathleen Sarmiento, ${ }^{11}$ Michelle Baladi, ${ }^{11}$ Patricia Chandler, ${ }^{11}$ Lawrence Lee, ${ }^{12}$ Atul Malhotra. ${ }^{1}$ University of Toronto, Toronto, Canada; ${ }^{2}$ Hephata Klinik, Schwalmstadt, Germany; ${ }^{3}$ Philipps University Marburg, Marburg, Germany; ${ }^{4}$ Grenoble Alpes University Hospital, Grenoble, France; ${ }^{5}$ University of Pennsylvania, Philadelphia, USA; ${ }^{6}$ Sahlgrenska University Hospital, Gothenburg University, Gothenburg, Sweden; ${ }^{7}$ Cleveland Sleep Research Center, Middleburg Heights, USA; ${ }^{8}$ Cleveland Clinic Lerner College of Medicine, Cleveland, USA; ${ }^{9}$ University of Pittsburgh/Veterans Administration Pittsburgh Health System, Pittsburgh, USA; ${ }^{10}$ San Francisco Veterans Administration Healthcare System, San Francisco, USA; ${ }^{11}$ Jazz Pharmaceuticals, Palo Alto, USA; ${ }^{12}$ Division of Pulmonary, Critical Care and Sleep Medicine, University of California San Diego, La Jolla, USA

10.1136/bmjresp-2019-bssconf.5

Introduction Solriamfetol (formerly JZP-110), a dopamine and norepinephrine reuptake inhibitor, has been approved in the United States to improve wakefulness in adults with excessive daytime sleepiness (EDS) associated with narcolepsy (75-150 $\mathrm{mg}$ ) or obstructive sleep apnoea (OSA; 37.5-150 mg). A Marketing Authorisation Application for these indications is under review with the European Medicines Agency. This study evaluated the long-term safety and efficacy of solriamfetol.

Methods Participants with EDS associated with narcolepsy or OSA who completed prior solriamfetol studies initiated open-label treatment with a 2 -week titration phase followed by a maintenance phase of $\leq 50$ weeks. A 2 -week, placebocontrolled, randomised withdrawal (RW) phase was conducted after 6 months. Change from beginning to end of the RW phase in Epworth Sleepiness Scale (ESS) was the primary endpoint; Patient and Clinician Global Impression of Change (PGI-C and CGI-C, respectively) were secondary endpoints.

Results Safety population comprised 643 participants (226 narcolepsy; 417 OSA); 280 participants (141 placebo; 139 solriamfetol) comprised the RW modified intent-to-treat population. A total of 458 participants $(71 \%)$ completed the study. Maintenance of efficacy in this 1-year study was demonstrated on the ESS, PGI-C, and CGI-C. Least squares mean change from the beginning to the end of the RW phase in ESS score was 5.3 versus 1.6 in participants randomised to placebo or solriamfetol, respectively $(\mathrm{P}<0.0001)$. Greater percentages of participants randomised to placebo

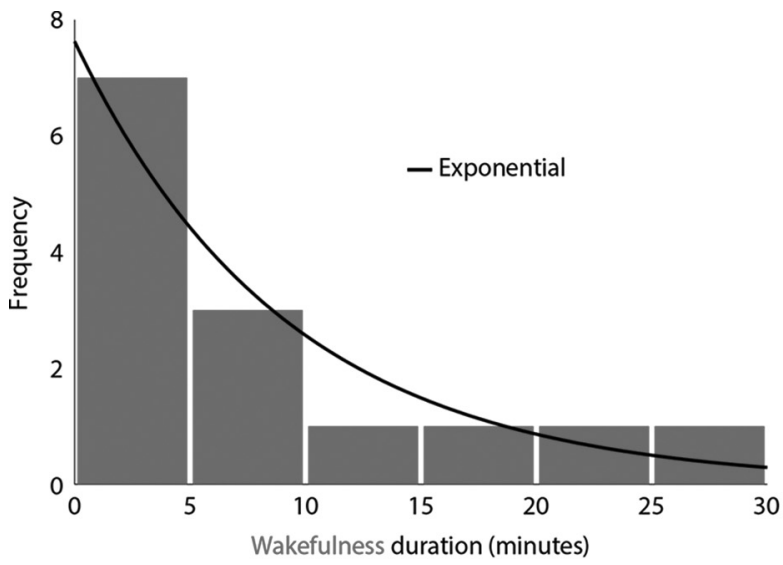

versus solriamfetol in the RW phase reported as worse on PGI-C and CGI-C (both $\mathrm{P}<0.0001$ ). The most frequent adverse events (AEs; $\geq 5 \%$ ) were headache, nausea, nasopharyngitis, insomnia, dry mouth, anxiety, decreased appetite, and upper respiratory tract infection; 27 (4.2\%) participants had $\geq 1$ serious AE.

Discussion These results demonstrate the long-term efficacy of solriamfetol for EDS in participants with narcolepsy or OSA. Safety profile following long-term administration was consistent with prior solriamfetol studies.

Support Jazz Pharmaceuticals.

\section{P006 MODELLING SLEEP-WAKE TRANSITIONS IN VERY AND MODERATELY PRE-TERM INFANTS}

${ }^{1}$ Anastasis Georgoulas, ${ }^{1}$ Asif Tamuri, ${ }^{2}$ Maria Pureza Laudiano-Dray, ${ }^{3}$ Judith Meek, ${ }^{2}$ Lorenzo Fabrizi, ${ }^{2}$ Kimberley Whitehead*. ${ }^{1}$ Research IT Services, University College London, London, UK; ${ }^{2}$ Dept. of Neuroscience, Physiology and Pharmacology, University College London, London, UK; ${ }^{3}$ Elizabeth Garrett Anderson Wing, University College London Hospitals, London, UK

\subsection{6/bmjresp-2019-bssconf.6}

Introduction Sleep is the dominant vigilance state in pre-term infants, but its regulation is still poorly understood, with no underpinning quantitative framework.

In animal studies, neonatal sleep-wake characteristics follow statistical patterns, e.g. wakefulness durations exhibit an exponential distribution. ${ }^{1}$ Here we investigated whether the same holds true for pre-term human infants.

Methods We recorded electroencephalography (EEG), respiratory movement, electrocardiography (ECG), and behavioural observations for up to two hours from 54 nonmechanically ventilated infants being cared for on the neonatal unit $(28+2-34+1$ weeks + days corrected gestational age). Data were staged as sleep or wakefulness in 30-s epochs (figure 1).

We characterised i) the distribution of wakefulness durations, using the Kolmogorov-Smirnov goodness-of-fit test, and ii) the likelihood of transitioning from sleep to wakefulness during the recording, using the Kaplan-Meier estimator which takes account of censored observations, i.e. when the event of interest (wakefulness) was not captured.

Results 14/54 (26\%) infants cycled through wakefulness during the recording: durations ranged from 2 to 29 minutes and

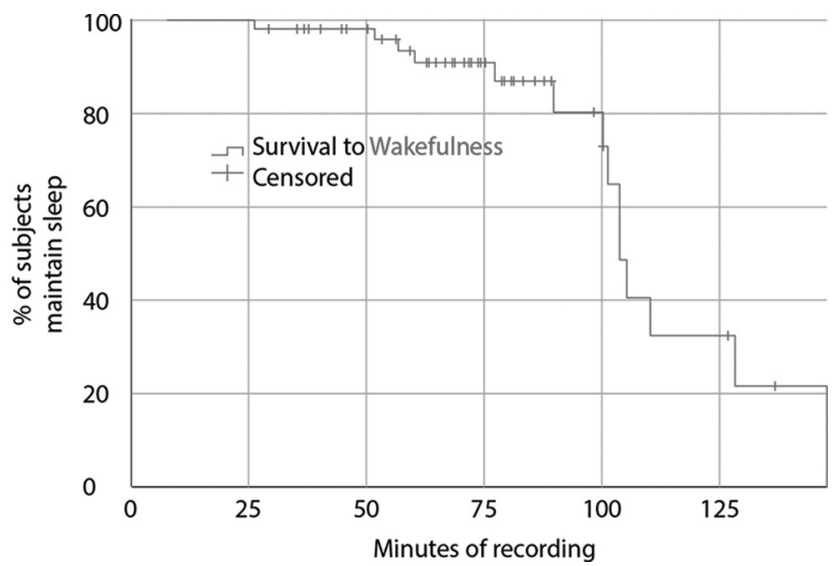

